\title{
Estudo da Curva de Crescimento de Animais da Raça Guzerá e seus Cruzamentos Alimentados a Pasto, com e sem Suplementação. 1. Análise e Seleção das Funções Não-Lineares ${ }^{1}$
}

\author{
Luís Orlindo Tedeschi², Celso Boin ${ }^{3}$, Romeu Fernandes Nardon ${ }^{4}$, Paulo Roberto Leme ${ }^{5}$
}

RESUMO - O objetivo deste trabalho foi analisar sete funções não-lineares para descrever o crescimento de machos e fêmeas da raça Guzerá e seus cruzamentos (3/4Guzerá-1/4Pardo Suíço, 1/2Nelore-1/4Pardo Suíço-1/4Guzerá, 1/2Pardo Suíço-1/2Guzerá, 1/2Chianina-1/4Pardo Suíço-1/4Guzerá, 1/2Caracú-1/2Guzerá, 1/2Caracu-1/4Pardo Suíço-1/4Guzerá) submetidos a três níveis de suplementação (sem, durante o período seco e o ano todo), a fim de analisar e selecionar uma função que apresentasse menores desvios de regressão, menor variação residual, maior coeficiente de determinação, menor variação entre os parâmetros dos animais, maior convergência e valor assintótico compatível com o peso adulto observado. A função que melhor se enquadrou nesses parâmetros de seleção foi a função de Gompertz, seguida da função de Von Bertalanffy. A função de Richards apresentou grande dificuldade computacional para o ajuste, resultando em baixa convergência. As funções de Brody apresentaram as maiores estimativas de peso adulto e a função de Gompertz modificada e Logística, as menores.

Palavras-chave: funções não-lineares, gado de corte

\section{Growth Curve Analysis of Guzera and Their Crossbreds Fed under Grazing with or without Supplementation. 1. Analysis and Selection of Nonlinear Functions}

ABSTRACT - The objective of this work was to evaluate seven non linear functions to describe Guzera male and female growth curves, and their crossbreds (3/4Guzera-1/4Brown Swiss, 1/2Nellore-1/4Brown Swiss-1/4Guzera, 1/2Guzera-1/2Brown Swiss, 1/2Chianina1/4Brown Swiss-1/4Guzera, 1/2Caracu-1/2Guzera, 1/2Caracu-1/4Brown Swiss-1/4Guzera) submitted to three levels of supplementation (without, during the dry period and the whole year), to analize and to select one function which presented lower regression deviation, lower residual sum of squares, higher coefficient of determination, lower variation among animal parameters, higher convergence and assintotic compatible value with the observed mature weight. The function that best express these parameters of selection was the Gompertz, followed by the Von Bertalanffy function. The Richards function showed the high computational difficulty to the adjustment, resulting on lower convergence. Brody functions presented the largest and the modified Gompertz and Logistic functions showed the smallest mature weight.

Key Words: beef cattle, nonlinear functions

\section{Introdução}

O conhecimento e o controle do crescimento e desenvolvimento dos ruminantes são tópicos de bastante interesse para os pesquisadores, pois o seu domínio permite que o manejo nutricional dos animais possa ser conduzido eficientemente (WEBSTER et al., 1982), além de permitir que programas de seleção animal sejam elaborados para as características de crescimento inerentes a cada raça (FITZHUGH, 1976).

Os parâmetros das funções que descrevem o crescimento dos animais são utilizados em modelos atuais de simulação para estimar a composição corporal do animal em qualquer ponto do crescimento, bastando apenas as informações de crescimento e composição corporal inicial do animal (KEELE et al., 1992; WILLIAMS et al., 1992), permitindo assim a predição das exigência de energia e proteína do animal.

Por intermédio dessas funções, podem-se selecionar animais que apresentam altas ou baixas taxas de crescimento relativo ao peso adulto (conhecidas como taxas de maturidade). Animais com maiores taxas de maturidade são mais precoces que animais que apresentam menores taxas de maturidade. Do ponto de vista de melhoramento, esse parâmetro é de fundamental importância (BROWN et al., 1972; FITZHUGH, 1976), pois animais de maior crescimento relativo podem ser selecionados.

\footnotetext{
1 Parte da Tese de Mestrado apresentada à ESALQ/USP

2 Estudante de PhD, Cornell University, Ithaca, NY. Bolsista do CNPq. E.mail: Iot1@cornell.edu

3 Professor Aposentado do Depto. de Zootecnia/ESALQ/USP. E.mail: celsoboin@picture.com.br

4 Instituto de Zootecnia - Nova Odessa. E.mail: nardon@izsp.br

5 Professor do Depto. de Zootecnia/FZEA/USP - E.mail: prleme@usp.br.
} 
Os ajustes dos dados peso-idade de cada animal ou grupo de animais permitem obter informações descritivas da presente curva de crescimento do animal estudado e/ou informações de prognósticos futuros para animais do mesmo grupo racial sob a mesma situação ambiental. Portanto, a função de crescimento que é utilizada para descrever o crescimento do animal tanto para fins de exigência nutricional, como para seleção genética, é de extrema importância.

Várias funções têm sido empregadas para moldarem o crescimento animal. MOORE (1985) apresentou uma função generalizada para estimar o peso de animais desde a fase embrionária até o peso adulto. Funções com componentes logarítmicos, inversos e exponenciais foram utilizadas por LISKI (1987), enquanto modelos multifásicos e modelos fatoriais foram elaborados por MENCHACA (1990, 1991a, 1991b, 1992) e MENCHACA et al. (1993), respectivamente. Entretanto, as funções que atualmente apresentam grande uso são as funções não-lineares com componentes exponenciais. Essas funções apresentam interpretação biológica do crescimento e são facilmente comparadas entre diferentes cenários de produção. A Tabela 1 mostra as funções não-lineares para descrever e predizer o crescimento de animais mais utilizadas.

A escolha da melhor função pela avaliação da análise de resíduos da variância não é a opção ideal, pois os dados do tipo longitudinais (todas as coletas de peso são efetuadas no mesmo animal) apresentam erros correlacionados entre as observações de uma idade para a outra. Esses erros são ocasionados, principalmente, pelas flutuações do peso vivo ao longo do tempo, devido a vários fatores que não são necessariamente refletidos na curva.

Um dos métodos recomendados para escolha da curva de ajuste é a avaliação do desvio entre o valor predito e o observado em determinadas idades (BROWN et al., 1976), entretanto, nem sempre o peso em tais idades estão à disposição. Os métodos mais empregados são: a menor soma de quadrados residuais (PEROTTO et al., 1992), desvios de regressão, coeficiente de determinação $\left(\mathrm{R}^{2}\right)$, porcentagem e dificuldade de convergência (BROWN et al., 1976), comportamento das curvas e avaliação dos parâmetros por comparação e avaliação gráfica das curvas (FITZHUGH, 1976).

De forma geral, experimentos têm relatado que a função de Richards apresenta melhor modelagem e a função de Brody apresenta boa modelagem apenas para idades acima de seis meses (BROWN et al., 1976; DeNISE e BRINKS, 1985). Outros autores relataram ainda que a função de Brody superestima o valor de peso adulto enquanto que a função Logística subestima-o quando comparadas com as funções de Richards e Von Bertalanffy (DUARTE, 1975; PEROTTO et al., 1992), embora exista uma padronização na utilização da função de Brody para bovinos (BROWN et al, 1972; DUARTE, 1975; SILVEIRA JR., 1976; e LUDWIG, 1977).

O objetivo deste estudo foi selecionar a função não-linear que melhor descrevesse o crescimento de animais Guzerá e seus cruzamentos com Pardo Suíço, Nelore, Chianina e Caracú, com base nos parâmetros de percentagem de convergência, soma de quadrado dos resíduos, desvios de regressão, coeficiente de determinação e estimativa do peso adulto.

\section{Material e Métodos}

Analisou-se o conjunto de dados obtidos pelo projeto IZ-14.012/78 - "Pastejo contínuo pós-desmama com e sem suplementação protéica nas secas", da Divisão de Bovinos de Corte do Instituto de Zootecnia/CPA/SAA.

Este experimento foi composto de dois anos bases consecutivos de nascimento (1977 e 1978, respectivamente, experimentos 1 e 2) para a coleta de dados de machos e fêmeas, durante aproximadamente três a quatro anos de idade. Estes animais são o resultado do cruzamento de fêmeas Guzerá (GU) com Pardo Suíço (PS), Nelore (NE), Chianina (CH) e Caracú (CA). A composição genética dos animais foi a seguinte: GU apenas, 3/4GU1/4PS, 1/2NE1/4PS1/4GU, 1/2PS1/ 2GU, 1/2CH1/4PS1/4GU, 1/2CA1/2GUe 1/2CA1/4PS1/ 4GU submetidos a três níveis de suplementação: sem suplementação (S1), suplementados apenas durante o período seco do ano (S2) e durante o ano todo (S3).

Para os animais que nasceram em 1977 (experimento 1), os cruzamentos GU, 3/4GU1/4PS, 1/2NE1/ 4PS1/4GU, 1/2PS1/2GU e 1/2CH1/4PS1/4GU foram submetidos aos seguintes tratamentos: sem e com suplementação apenas "nas secas" de $0,5 \mathrm{~kg}$ de farelo de soja por animal por dia. O total de animais utilizados para essa análise foi de 61 machos e 58 fêmeas. Este experimento foi designado de ano base 1978, pois a suplementação teve início nesse ano.

Para os animais que nasceram em 1978 (experimento 2), todos os cruzamentos foram avaliados e os animais foram suplementados com uma ração à base de $69,1 \%$ de farelo de trigo, $29,6 \%$ de milho e $1,3 \%$ de uréia (base seca), recebendo $1,5 \mathrm{~kg}$ por animal por 
632 Rev. bras. zootec.

dia. Todos os grupos raciais foram submetidos aos níveis de suplementação sem e com suplementação apenas durante o período seco do ano. Apenas os grupos raciais GU, 3/4GU1/4PS e 1/2NE1/4PS1/ 4GU receberam a suplementação durante o ano todo. O total de animais utilizados para análise foi de 90 machos e 89 fêmeas. Este experimento foi designado de ano base 1979.

Os animais foram manejados em pastagem de colonião em sistema de pastejo contínuo na Estação Experimental de Zootecnia de Andradina/SP e no Posto Experimental de Castilho/SP. A suplementação foi fornecida apenas durante o período matutino. As pesagens foram efetuados a cada 28 dias, com animais em jejum de 18 horas de água e alimento. Todos os animais foram periodicamente vacinados, vermifugados e banhados contra carrapatos.

Foi ainda considerada correção de peso vivo em jejum das fêmeas que se apresentavam prenhas. Dessa forma, foram avaliados os machos e as fêmeas sem $(\mathrm{F})$, com $\left(\mathrm{F}_{\mathrm{aj}}\right)$ ajuste do peso vivo para o peso fetal e tecidos anexos por intermédio das equações empíricas de estimativa de peso total do útero proposto por PRIOR e LASTER (1979).

A obtenção dos parâmetros das funções nãolineares foi realizada por meio do procedimento NLIN do software SAS (1987), utilizando-se o método Gauss-Newton para a convergência dos dados (HARTLEY, 1961; LAWTON et al., 1972). Os procedimentos delineados por PEREIRA e ARRUDA (1987) e SAS (1987) foram utilizados para convergirem os dados às funções não-lineares. Os valores das somas de quadrados do resíduo (SQr), coeficiente de determinação $\left(\mathrm{R}^{2}\right)$ e desvios de regressão de cada função esquematizada na Tabela 1 foram utilizados para selecionar uma função para descrever o crescimento dos animais. A comparação destes valores entre as funções não-lineares utilizadas foi realizada por intermédio do seguinte modelo estatístico:

$$
Y_{i j}=\mu+\text { Função }_{i}+\varepsilon_{i j}
$$

O teste de REGWF (Ryan-Einot-Gabriel-Welsh F, SAS, 1987) foi utilizado nas comparações múltiplas em nível de 5\%. A análise multivariada de agrupamento foi efetuada para comparar as funções não-lineares ao mesmo tempo para vários fatores. Neste caso, o procedimento CLUSTER (SAS, 1987) foi utilizado.

\section{Resultados e Discussão}

O parâmetro " $A$ ", das funções da Tabela 1, representa o peso adulto, quando o tempo (" $t$ ") tende
Tabela 1 - Funções não-lineares utilizadas para descreveram o crescimento animal

Table 1 - Nonlinear functions used to describe animal growth curves

\begin{tabular}{|c|c|}
\hline $\begin{array}{l}\text { Nome } \\
\text { Name }\end{array}$ & $\begin{array}{l}\text { Função } \\
\text { Function }\end{array}$ \\
\hline Brody $^{1}$ & A. $\left(1-b \cdot e^{-k \cdot t}\right)$ \\
\hline Brody modificada $^{2}$ & $\mathrm{~A}-(\mathrm{A}-\mathrm{PN}) \cdot \mathrm{e}^{(-\mathrm{k} \cdot \mathrm{t})}$ \\
\hline $\begin{array}{l}\text { Modified Brody } \\
\text { Von Bertalanffy }\end{array}$ & A. $\left(1-b \cdot e^{-k \cdot t}\right)^{3}$ \\
\hline Logística $^{4}$ & A. $\left(1+b \cdot e^{-k \cdot t}\right)^{-1}$ \\
\hline $\begin{array}{l}\text { Logistic } \\
\text { Gompertz }\end{array}$ & A. $e^{\left(-b \cdot e^{-k \cdot t}\right)}$ \\
\hline $\begin{array}{l}\text { Gompertz modificada } \\
\text { Modified Gompertz }\end{array}$ & $P N \cdot e^{d .\left(1-e^{(-k . t)}\right)}$ \\
\hline Richards 6 & A. $\left(1-b \cdot e^{-k \cdot t}\right)^{m}$ \\
\hline
\end{tabular}

${ }^{1}$ BRODY (1945).

${ }^{2} \mathrm{CSIRO}(1990)$.

3VON BERTALANFFY (1938).

${ }^{4}$ FITZHUGH (1976).

5JOANDERT E CARTWRIGHT (1969).

${ }^{6}$ RICHARDS (1959).

A: Peso adulto (Mature weight), b: Coeficiente de integração (Integration factor), k: Taxa de maturidade (maturing rate), t: Tempo (time), $\mathrm{m}$ : Coeficiente de inflexão (inflection factor), PN: Peso ao nascer (Birth weight).

ao infinito. O parâmetro " $b$ " é o fator de integração que ajusta os valores de peso inicial e geralmente está associado com o peso ao nascer. O parâmetro " $k$ ", taxa de maturidade, é uma função entre a máxima taxa de crescimento e o peso adulto do animal. Quando maior esse valor, mais precoce será o animal em termos de crescimento, pois atingirá o peso adulto em menor tempo. Finalmente, o parâmetro " $m$ " refere-se ao ponto de inflexão da função de Richards (FITZHUGH, 1976). Nota-se que " $m$ " equivale a $1 \mathrm{e}$ 3 , respectivamente, nas funções de Brody e Von Bertalanffy.

Uma análise de sensibilidade no valor do peso adulto foi efetuada no sentido de mostrar a capacidade de convergência de cada função e a tendência média do valor da estimativa do peso adulto. A Tabela 2 mostra as porcentagens de animais que convergiram e a estimativa de peso adulto (" $A$ ") foi inferior ao peso adulto determinado. A mesma análise foi efetuada para as fêmeas com e sem correção de peso (Tabelas 4 e 3 , respectivamente).

Para os machos, observou-se a existência de dois grupos de funções para convergência: o primeiro grupo foi composto pelas funções de Brody modificada, Brody e Richards, que apresentaram baixa convergência e tendência de maior estimativa de peso adulto. $\mathrm{O}$ segundo grupo foi composto pelas demais funções, apresentando maioralta convergência e tendência de menor estimativa de peso adulto (Tabela 2). Por 
TEDESCHI et al.

Tabela 2 - Porcentagem de convergência dos machos para cada função não-linear, conforme determinado valor de peso adulto

Table 2 - Percentage of convergence of nonlinear functions for males according to a mature weight value

\begin{tabular}{|c|c|c|c|c|c|c|c|c|c|c|c|c|}
\hline \multirow[t]{2}{*}{$\begin{array}{l}\text { Função } \\
\text { Function }\end{array}$} & \multicolumn{12}{|c|}{$\begin{array}{l}\text { Peso adulto } \\
\text { Mature weight }\end{array}$} \\
\hline & 500 & 550 & 600 & 650 & 700 & 750 & 800 & 850 & 900 & 950 & 1000 & 1050 \\
\hline Brody_M & 1,9 & 3,3 & 4,6 & 9,3 & 12,6 & 17,9 & 25,8 & 37,8 & 45,7 & 56,9 & 62,3 & 67,6 \\
\hline Brody & 0,6 & 2,6 & 3,9 & 5,3 & 9,2 & 11,2 & 13,9 & 19,2 & 27,8 & 32,5 & 37,8 & 43,7 \\
\hline Logística & 18,5 & 41,1 & 76,1 & 90,1 & 94,0 & 96,0 & 97,4 & 97,3 & 98,7 & 99,4 & 99,3 & 99,3 \\
\hline Gompertz & 5,9 & 16,5 & 34,4 & 59,6 & 77,5 & 86,1 & 90,7 & 92,7 & 94,7 & 96,0 & 96,0 & 96,7 \\
\hline Bertalanffy & 3,9 & 10,6 & 18,5 & 37,1 & 55,6 & 73,5 & 78,1 & 84,8 & 88,1 & 90,7 & 91,4 & 92,7 \\
\hline Richards & 0 & 0 & 0,7 & 0,7 & 1,9 & 2,7 & 2,7 & 2,7 & 3,3 & 3,9 & 4,6 & 5,9 \\
\hline Gomp_M & 45,7 & 72,2 & 96,0 & 100 & 100 & 100 & 100 & 100 & 100 & 100 & 100 & 100 \\
\hline
\end{tabular}

Brody_M: Brody modificada (Modified Brody function), Gomp_M: Gompertz modificada (Modified Gompertz function).

Tabela 3 - Porcentagem de convergência das fêmeas sem correção de peso fetal e tecidos anexos da placenta para cada função não-linear, conforme determinado valor de peso adulto

Table 3 - Percentage of convergence of nonlinear functions for females without body weight adjustments for fetal tissues according to a mature weight value

\begin{tabular}{|c|c|c|c|c|c|c|c|c|c|c|c|c|}
\hline \multirow[t]{2}{*}{$\begin{array}{l}\text { Função } \\
\text { Function }\end{array}$} & \multicolumn{12}{|c|}{$\begin{array}{l}\text { Peso adulto } \\
\text { Mature weight }\end{array}$} \\
\hline & 500 & 550 & 600 & 650 & 700 & 750 & 800 & 850 & 900 & 950 & 1000 & 1050 \\
\hline Brody_M & 40,8 & 56,4 & 72,7 & 77,5 & 81,6 & 86,3 & 88,4 & 91,1 & 93,2 & 93,2 & 93,2 & 95,9 \\
\hline Brody & 46,9 & 60,5 & 74,1 & 78,9 & 82,9 & 86,3 & 89,1 & 91,8 & 92,5 & 93,2 & 93,8 & 94,5 \\
\hline Logística & 87,0 & 93,8 & 95,9 & 97,2 & 97,9 & 97,9 & 98,6 & 99,3 & 99,3 & 100 & 100 & 100 \\
\hline Gompertz & 74,1 & 87,0 & 93,8 & 95,2 & 95,9 & 95,9 & 97,2 & 97,9 & 97,9 & 97,9 & 98,6 & 98,6 \\
\hline Bertalanffy & 68,7 & 84,3 & 89,1 & 93,8 & 95,2 & 95,2 & 95,9 & 95,9 & 97,2 & 97,2 & 97,9 & 97,9 \\
\hline Richards & 12,2 & 17,0 & 18,3 & 19,0 & 20,4 & 20,4 & 21,0 & 21,0 & 21,7 & 21,7 & 21,7 & 21,7 \\
\hline Gomp_M & 87,7 & 95,9 & 97,9 & 99,3 & 100 & 100 & 100 & 100 & 100 & 100 & 100 & 100 \\
\hline
\end{tabular}

Brody_M: Brody modificada (Modified Brody function), Gomp_M: Gompertz modificada (Modified Gompertz function).

Tabela 4 - Porcentagem de convergência das fêmeas com correção de peso fetal e tecidos anexos da placenta para cada função não-linear, conforme determinado valor de peso adulto

Table 4 - Percentage of convergence of nonlinear functions for females with body weight adjustments for fetal tissues according to a mature weight value

\begin{tabular}{|c|c|c|c|c|c|c|c|c|c|c|c|c|}
\hline \multirow[t]{2}{*}{$\begin{array}{l}\text { Função } \\
\text { Function }\end{array}$} & \multicolumn{12}{|c|}{$\begin{array}{l}\text { Peso adulto } \\
\text { Mature weight }\end{array}$} \\
\hline & 500 & 550 & 600 & 650 & 700 & 750 & 800 & 850 & 900 & 950 & 1000 & 1050 \\
\hline Brody_M & 53,0 & 69,3 & 78,2 & 83,6 & 87,0 & 88,4 & 90,4 & 91,8 & 93,2 & 93,2 & 93,2 & 95,9 \\
\hline Brody & 55,1 & 70,7 & 78,9 & 82,9 & 87,7 & 89,1 & 89,8 & 93,2 & 93,8 & 93,8 & 94,5 & 94,5 \\
\hline Logística & 88,4 & 94,5 & 95,9 & 97,2 & 97,9 & 97,9 & 98,6 & 99,3 & 99,3 & 100 & 100 & 100 \\
\hline Gompertz & 82,3 & 90,4 & 93,8 & 95,2 & 95,9 & 95,9 & 97,2 & 97,9 & 97,9 & 97,9 & 98,6 & 98,6 \\
\hline Bertalanffy & 76,8 & 87,0 & 91,8 & 93,8 & 95,2 & 95,2 & 95,9 & 95,9 & 97,2 & 97,2 & 97,9 & 97,9 \\
\hline Richards & 17,6 & 25,1 & 25,8 & 26,5 & 27,2 & 27,2 & 27,8 & 27,8 & 28,5 & 28,5 & 28,5 & 28,5 \\
\hline Gomp_M & 91,1 & 96,6 & 97,9 & 99,3 & 100 & 100 & 100 & 100 & 100 & 100 & 100 & 100 \\
\hline
\end{tabular}

Brody_M: Brody modificada (Modified Brody function), Gomp_M: Gompertz modificada (Modified Gompertz function).

exemplo, para a função logística, $90 \%$ dos animais apresentaram peso adulto inferior a $650 \mathrm{~kg}$, ao passo que, para a função de Brody, a porcentagem de convergência foi 10 vezes menor $(9 \%)$.

Para as fêmeas sem correção de peso (Tabela 3), pode-se notar que a percentagem de convergência foi maior em relação aos machos (Tabela 2). Foram identificados também três grupos de convergência: o primeiro composto pelas funções Logística, Gompertz, Von Bertalanffy e Gompertz modificada que apresentaram altas taxas de convergência; o segundo grupo composto pelas funções de Brody modificada e de Brody, com altas taxas de convergência apenas para peso adulto elevado e, finalmente, a função de Richards, correspondente ao terceiro grupo, o qual não apresentou convergência satisfatória. 
634 Rev. bras. zootec.

A Tabela 4 mostra a porcentagem de convergência para as fêmeas com correção de peso vivo. Quando comparado com a Tabela 3, pode-se notar que existiu maior facilidade de convergência para todas as curvas, provavelmente devido à menor flutuação do peso vivo em jejum causada pelos pesos dos tecidos anexos à placenta e ao feto. Analisandose estas tabelas, notou-se que a correção para o peso vivo influenciou a taxa de convergência e também diminuiu proporcionalmente a estimativa de peso adulto dos animais, confirmando os dados de FITZHUGH (1976). A baixa convergência entre os machos (Tabela 3) foi causada principalmente pela idade de abate dos animais. Isso significa que, à idade de abate, os animais não apresentaram constância de peso em jejum, pois ainda estavam na fase de crescimento. Dados de animais em crescimento a pasto têm indicado que, dependendo da qualidade nutricional da forragem e de técnicas de suplementação estratégica (TEDESCHI et al., 2000), o peso adulto somente será atingido após 4 a 5 anos de idade. MORROW et al. (1978), analisando animais da raça Angus (tamanho corporal pequeno), mostraram que a estabilidade de peso foi obtida somente após 4,5 anos de idade. De forma semelhante, BELTRAN et al. (1992) constataram que o peso se manteve constante após 5,5 anos de idade. Estes dados indicam que animais de idade menor que 4,5 anos dificilmente terão convergência satisfatória nas funções não-lineares para crescimento, pois ainda não atingiram o tamanho corporal adulto.

Após a obtenção dos parâmetros inerentes a cada função para cada animal analisado, foi realizada uma seleção dos animais que convergiram e obtiveram peso adulto (" $A$ ") inferior a $1000 \mathrm{~kg}$. Esse valor limite é um valor aceitável biologicamente e condiz com dados publicados de peso adulto alcançados por animais de mesmo grau de estrutura corporal que os animais deste experimento (MENCHACA et al., 1996).

A Tabela 5 mostra os quatro índices que foram utilizados para a seleção da função que melhor descrevia os dados de crescimento dos animais. A estimativa de peso adulto apresentou interação com vários fatores; portanto, o valor médio utilizado na Tabela 5 serve apenas para comparação entre funções e, por conseguinte, não deve ser utilizado como padrão para cálculos de curvas de crescimento.

As funções Logística, Gompertz, Von Bertalanffy e Gompertz modificada apresentaram os menores valores somas de quadrados do resíduo, porém, somente as funções de Gompertz e de Von Bertalanffy diferiram $(\mathrm{P}<0,05)$ das funções de Brody modificada, Brody e Richards; as demais diferiram $(\mathrm{P}<0,05)$ apenas da Richards. O coeficiente de determinação $\left(\mathrm{R}^{2}\right)$ foi maior para as funções Logística, Gompertz, Von Bertalanffy e Gompertz modificada $(\mathrm{P}<0,05)$. Para os desvios da regressão, as funções de Brody modificada, Logística, Gompertz e Von Bertalanffy apresentaram os menores valores $(\mathrm{P}>0,05)$. As funções de Gompertz e Von Bertalanffy apresentaram os valores peso adulto mais próximos às médias de todos, os quais estão bem próximo de valor encontrados na literatura (MENCHACA et al., 1996) e seguem os dados observados dos animais estudados.

A Tabela 6 mostra os desvios médios de regressão de alguns pontos específicos da curva de crescimento de fêmeas sem (F) e com ( $\left.F_{a j}\right)$ correção de peso fetal. Em geral, notou-se que os pesos ao nascer, aos 40 e 50 meses foram superestimados pelas funções, ao passo que o peso à desmama ( \pm 250 dias) e os pesos aos 20 , 30 e 45 meses foram subestimados.

Tabela 5 - Médias ajustadas dos fatores para a seleção da função de crescimento Table 5 - Adjusted means of some parameters for growth curve selection

\begin{tabular}{lcccccccc}
\hline & $\begin{array}{c}\text { SQ resíduo } \\
\text { Error SS }\end{array}$ & R & \multicolumn{3}{c}{$\begin{array}{c}\text { Desvios } \\
\text { Deviations }\end{array}$} \\
\hline Brody_M & 28298 & bc & 91,22 & b & 27,20 & bcd & 596,34 & a \\
Brody & 29861 & b & 90,94 & b & 28,10 & b & 571,03 & b \\
Logística & 24957 & cd & 92,23 & a & 27,05 & bcd & 482,57 & ef \\
Gompertz & 24233 & d & 92,50 & a & 26,17 & cd & 515,81 & cd \\
Bertalanffy & 24330 & d & 92,50 & a & 25,97 & d & 536,83 & c \\
Richards & 55880 & a & 82,98 & b & 39,16 & a & 493,18 & de \\
Gomp_M & 27157 & bcd & 91,59 & ab & 27,79 & bc & 462,12 & f
\end{tabular}

1 Desvios de regressão (Regression deviations).

Brody M: Brody modificada (Modified Brody function), Gomp M: Gompertz modificada (Modified Gompertz function).

Letras distintas na mesma coluna indicam diferença $(P<0,05)$ pelo teste REGWF.

Different letters within column are different $(P<.05)$ by REGWF test. 
TEDESCHI et al.

Tabela 6 - Menores desvios de regressão em alguns pontos das curvas de crescimento das fêmeas 1 Table 6 - Lower regression deviation of female growth curves in some selected points

\begin{tabular}{|c|c|c|c|c|c|c|c|c|}
\hline \multirow[t]{2}{*}{$\begin{array}{l}\text { Ano } \\
\text { Year }\end{array}$} & \multirow[t]{2}{*}{$\begin{array}{l}\text { Sexo } \\
\text { Sex }\end{array}$} & \multicolumn{6}{|c|}{$\begin{array}{c}\text { Idade (meses) } \\
\text { Age (month) }\end{array}$} & \multirow[b]{2}{*}{$(+) 50$} \\
\hline & & (+) 7 & $(-) 9$ & $(-) 20$ & $(-) 30$ & $(+) 40$ & (-) 45 & \\
\hline 1 & $\mathrm{~F}$ & $\begin{array}{l}\mathrm{Bm}^{* * *} \\
(0)\end{array}$ & $\begin{array}{l}\mathrm{Bm}^{*} \\
(20,9)\end{array}$ & $\begin{array}{c}\mathrm{Ri} \\
(6,7)\end{array}$ & $\begin{array}{c}\text { Lo } \\
(42,2)\end{array}$ & $\begin{array}{c}\mathrm{Gm} \\
(22,9)\end{array}$ & $\begin{array}{c}\mathrm{Bm} \\
(20,9)\end{array}$ & $\begin{array}{c}\mathrm{Gm} \\
(27,0)\end{array}$ \\
\hline & $\mathrm{F}_{\mathrm{aj}}$ & $\begin{array}{c}\mathrm{Bm}^{* * *} \\
(0)\end{array}$ & $\begin{array}{l}\mathrm{Bm}^{* * * *} \\
(20,3)\end{array}$ & $\begin{array}{c}\mathrm{Ri} \\
(9,0)\end{array}$ & $\begin{array}{c}\mathrm{Ri} \\
(32,0)\end{array}$ & $\begin{array}{c}\mathrm{Gm}^{*} \\
(16,7)\end{array}$ & $\begin{array}{c}\mathrm{Bm} \\
(23,5)\end{array}$ & $\begin{array}{c}\mathrm{Gm} \\
(24,9)\end{array}$ \\
\hline 2 & $\mathrm{~F}_{\mathrm{aj}}$ & $\begin{array}{c}\mathrm{Bm}^{* * *} \\
(0) \\
\mathrm{Bm}^{* * *} \\
(0)\end{array}$ & $\begin{array}{c}\mathrm{Bm}^{*} \\
(12,5) \\
\mathrm{Bo}^{*} \\
(12,3)\end{array}$ & $\begin{array}{c}\text { Go } \\
(6,6) \\
\text { Lo } \\
(6,6)\end{array}$ & $\begin{array}{c}\mathrm{Ri} \\
(24,9) \\
\mathrm{Lo} \\
(23,8)\end{array}$ & $\begin{array}{c}\mathrm{Ri} \\
(13,0) \\
\mathrm{Gm} \\
(12,7)\end{array}$ & & \\
\hline
\end{tabular}

1 Os sinais de (+) e (-) significam, respectivamente, super e subestimativa do peso vivo pelas funções em relação ao valor médio observado. Os sinais de ${ }^{* * *} e^{*}$ indicam diferenças estatísticas em nível de 0,1 e $5 \%$, respectivamente, pelo LSMEANS entre as funções. As funções com o menor desvio são apresentados como Brody modificada (Bm), Richards (Ri), Logística (Lo), Gompertz modificada (Gm), Gompertz (Go) e Brody (Bo).

The signs (+) and (-) indicate over- or underprediction of body weight in some selected points by growth curves when compared to the observed body weight. The symbols ${ }^{* * *}$ and ${ }^{*}$ indicate statistical difference by LSMeans respectively to $0.1 \%$ and $5 \%$ between functions. The function of lowest regression deviation value is shown as modified Brody (Bm), Richards (Ri), Logistic (Lo), modified Gompertz (Gm), Gompertz (Go), or Brody (Bo).

Ainda na Tabela 6, constata-se que não houve diferença entre os desvios das funções, a partir da desmama (acima de 9 meses). Apenas a função de Brody modificada ajustou melhor antes desse ponto, devido à sua estrutura matemática, que ajusta eficientemente o peso no tempo inicial (ao nascer), mas, em contra-partida, tende a superestimar opeso adulto(COMMONWEALTH SCIENTIFIC AND INDUSTRIAL RESEARCH ORGANISATION - CSIRO, 1990). Os desvios para as fêmeas com correção para peso vivo são menores em relação às das fêmeas sem correção.

Com a análise multivariada de agrupamento (distância Euclidiana) das funções, foi possível agrupar as funções semelhantes em relação à soma de quadrado do resíduo, desvios da regressão, coeficiente de determinação, peso adulto e taxa de maturidade.

O resultado deste agrupamento multivariado mostrou que as funções Von Bertalanffy e Richards, Brody e Brody modificada, Gompertz e Gompertz modificada e Gompertz modificada e Logística apresentaram comportamentos semelhantes. Quando apenas o peso adulto e a taxa de maturidade foram analisados, as funções semelhantes, em ordem de significância, foram: Von Bertalanffy e Gompertz, Brody modificada e Gompertz, Brody e Gompertz, Brody e Brody modificada, Gompertz modificada e Richards e Logística e Richards. Estes resultados estão de acordo com as análises de semelhanças entre funções realizadas por BROWN et al. (1976) e FITZHUGH (1976).

Altas correlações entre coeficientes é indicativo de que os parâmetros estão medindo o mesmo efeito de crescimento do animal, conforme discutido por FITZHUGH (1976). Complementares à análise de agrupamento, as funções de Von Bertalanffy e Gompertz apresentaram alta correlações tanto para peso adulto $(0,99)$, como para taxa de maturidade $(0,99)$. As funções de Brody e Brody modificada apresentaram, também, altas correlações entre o peso adulto $(0,96)$ e a taxa de maturidade $(0,97)$. As funções de Gompertz e Logística também apresentaram altas correlações entre o peso adulto $(0,98)$ e a taxa de maturidade $(0,98)$. A função de Gompertz e Gompertz modificada não apresentaram altas correlações entre os seus parâmetros, sendo que essa última não teve nenhum tipo de correlação com o seu parâmetro " $d$ " (Tabela 1), mostrando que este parâmetro mede efeitos distintos em relação aos outros parâmetros das demais funções, devendo-se considerar as comparações entre funções não-lineares.

A função de Gompertz foi s que melhor se adaptou aos dados desses animais, tendo como base os fatores que foram avaliados e as informações prévias de literatura. As estimativas de peso adulto para as fêmeas Guzerá com suplementação durante o período das secas foi de 422 e $455 \mathrm{~kg}$, respectivamente, para os anos de 1978 e 1979. Estes valores estão próximos aos valores de $441 \mathrm{~kg}$, obtidos por PEROTTO et al. (1997), utilizando a função de Richards.

A comparação entre a média de cada parâmetro da curva de Gompertz, calculado para os animais individualmente, com o valor do parâmetro médio, 
calculado para os animais de mesmo grupo de interesse (média dos pesos), mostrou que, para a estimativa do peso adulto, o parâmetro médio obtido por intermédio de ajuste de todos os animais foi inferior ao valor médio de cada animal (484 e 526; $\mathrm{P}<0,01$ ); para o parâmetro " $b$ " não houve diferença estatística $(2,2741$ e 2,2695) e para a taxa de maturidade houve diferença estatística $(0,0030$ e 0,$0027 ; \mathrm{P}<0,01)$, conforme discutido por MERRELL (1931), indicando que comparações de pesos adultos e/ou taxas de maturidade estimados por funções não-lineares devem ser efetuadas utilizando-se o mesmo método de obtenção dos parâmetros.

\section{Conclusões}

O nível de dificuldade de convergência foi maior para a curva de Richards. As funções de Brody tenderam a superestimar o peso adulto, enquanto as funções de Gompertz modificada e Logística tenderam a subestimá-lo. A função selecionada por intermédio dos índices de comparação entre as funções estudadas, foi a de Gompertz. Os machos não apresentaram idade suficiente para a obtenção de ajuste adequado, devido ao fato de a idade de abate ser anterior à estabilização do crescimento (maturidade). As fêmeas com correção do peso vivo tenderam a mostrar menor desvio entre os valores estimados e os observados. Existiu diferença entre os parâmetros médios e a média dos parâmetros calculados para cada grupo de animais e para cada animal.

\section{Agradecimento}

Aos auxiliares técnicos José Carlos M. Rasteiro, Mitsuharu Kuriama, Sebastião L. Camargo, Maria H. Camargo e Valfrido V. Lemos, aos campeiros Deraldo Manoel, Elízio Alves e Adércio Palomares e aos tratadores Pedro Daniele Sebastião F. do Amaral, pela importante ajuda na condução deste experimento.

\section{Referências Bibliográficas}

BELTRAN, J.J., BUTTS,W.T., OLSON,T.A. et al. 1992. Growth patterns of two lines of Angus cattle selected using predicted growth parameters. J. Anim. Sci., 70(3):734-741.

BRODY, S. 1945. Bioenergetics and growth. New York: Reinhold Pub. 1023p.

BROWN, J.E., BROWN, C.J., BUTTS, W.T. 1972. A discussion of the genetic aspects of weight, mature weight and rate of maturing in Hereford and Angus cattle. J. Anim. Sci., 34:525-32.

BROWN, J.E., FITZHUGH JR., H.A., CARTWRIGHT,T.C. 1976. A comparison of nonlinear models for describing weight- age relationships in cattle. J. Anim. Sci., 42(4):810-818.

COMMONWEALTH SCIENTIFIC AND INDUSTRIAL RESEARCH ORGANISATION. 1990. Feeding standards for Australian livestock: ruminants. Melbourne: CSIRO. 266p.

DeNISE, R.S.K., BRINKS, J.S. 1985. Genetic and environmental aspects of the growth curve parameters in beef cows. J. Anim. Sci., 61(6):1431-1440.

DUARTE, F.A.M. Estudo da curva de crescimento de animais da raça Nelore (Bos taurus indicus) através de cinco modelos estocásticos. Ribeirão Preto, 1975. 284p. Tese (LivreDocência) - Faculdade de Medicina de Ribeirão Preto, Univeridade de São Paulo.

FITZHUGH, H.A. 1976. Analysis of growth curves and strategies for altering their shapes. J. Anim. Sci., 42(4):1036-1051.

HARTLEY, H.O. 1961. The modified Gauss-Newton method for the fitting of nonlinear regression functions by least squares. Technometric, 3(2):269-280.

JOANDERT, G.E., CARTWRIGHT, T.C. 1969. Estimation of efficiency of beef production. J. Anim. Sci., 29:862-868.

KEELE, J.W., WILLIAMS, C.B., BENNETT, G.L. 1992. A computer model to predict the effects of level of nutrition on composition of empty body gain in beef cattle. I. Theory and development. J. Anim. Sci., 70:841-857.

LAWTON, W.H., SYLVESTRE, E.A., MAGGIO, S. 1972. Self modeling nonlinear regression. Techinometrics, 14(30):513-532.

LISKI, E.P. 1987. A growth curve analysis for bulls tested at station. Biometrical J., 29(3):331-343.

LUDWIG, A. Ajustamento de curvas exponenciais ao crescimento de gado Nelore e análise de seus parâmetros. Viçosa: UFV, 1977. 84p. Dissertação (Mestrado) - Universidade Federal de Viçosa, 1977.

MENCHACA, M.A. 1990. The use of stage models for describing animal growth curves. Cuban J. Agric. Sci., 24(1):31-36.

MENCHACA, M.A. 1991a. Modelling of the bovine weight growth. 1. An intrinsically linear model for growth representation. Cuban J. Agric. Sci., 25(2):125-127.

MENCHACA, M.A. 1991b. Modelling of the bovine weight growth. 2. Multiplicative model controlling the growth curve and other effects. Cuban J. Agric. Sci., 25(5):494-501.

MENCHACA, M.A. 1992. Modelling of the bovine weight growth. 3. Growth stage-multiplicative model. Cuban J. Agric. Sci., 26(2):105-109.

MENCHACA, M.A., CHASE, C.C., OLSON, T.A. et al. 1996. Evaluation of growth curves of Brahman cattle of various frame sizes. J. Anim. Sci., 74(9):2140-2151.

MENCHACA, M.A., VALDES, G., BRITO, M. 1993. A study on the performance of grazing animals through the use of a growth multiplicative model. Cuban J. Agric. Sci., 27(1):11-6.

MERRELL, M. 1931. The relationship of individual growth to average growth. Human Biology, (3):37-70.

MOORE, A.J. 1985. A mathematical equation for animal growth from embryo to adult. Anim. Prod., 40(3):441-453.

MORROW, R.E., McLAREN, J.B., BUTTS, W.T. 1978. Effects of age on estimates of bovine growth-curve parameters. $J$. Anim. Sci., 47(2):352-357.

PEREIRA, A.R., ARRUDA, H.V. 1987. Ajuste prático de curvas na pesquisa biológica. Campinas: Fundação Cargill. 50p.

PEROTTO, D., CUE, R.I., LEE, A.J. 1992. Comparison of nonlinear functions for describing the growth curve of three genotypes of dairy cattle. Can. J. Anim. Sci., 72(4):773-782.

PEROTTO, D., CASTANHO, M.J.P., ROCHA, J.L. et al. 1997. Descrição das curvas de crescimento de fêmeas bovinas Guzerá, Gir, Holandês x Guzerá e Holandês x Gir. R. Bras. Zootec., 26(2):283-288. 
PRIOR, R.L., LASTER, D.B. 1979. Development of the bovine fetus. J. Anim. Sci., 48(6):1546-1553.

RICHARDS, F.J. 1959. A flexible growth function for empirical use. J. Exper. Botany, 10(20):290-300.

SAS INSTITUTE. 1987. SAS/STATTM Guide for personal computers. 6.ed. Cary. 1028p.

SILVEIRA JR., P. Estudos de alguns modelos experimentais no crescimento de bovinos da raça Ibajé. Piracicaba, 1976. 174p. Dissertação (Mestrado) - Escola Superior de Agricultura "Luiz de Queiroz", Universidade de São Paulo, 1976.

TEDESCHI, L.O., BOIN, C., NARDON, R.F. et al. 2000. Efeito da suplementação com concentrado no ganho de peso de bovinos da raça Guzerá e de seus cruzamentos, manejados em pastagens. Bol. Ind. Anim., 56(2):195-205.
VON BERTALANFFY, L. 1938. A quantitative theory of organic growth. Human Biology, (10):181-213.

WEBSTER, A.J.F., AHMED, A.A.M., FRAPPELL, J.P. 1982. A note of growth rates and maturation rates in beef bulls. Anim. Prod., 35(2):281-286.

WILLIAMS, C.B., KEELE, J.W., BENNETT, G.L.A. 1992. Computer model to predict the effects of level of nutrition on composition of empty body gain in beef cattle. II. Evaluation of the model. J. Anim. Sci., 70:858-866.

Recebido em: 19/02/99

Aceito em: 10/09/99 